\title{
ACCIDENTES POR MORDEDURA CANINA, CASOS REGISTRADOS EN UN HOSPITAL DE NIÑOS DE LIMA, PERÚ 1995 - 2009
}

\author{
Carmen Morales $1, a$, Néstor Falcón ${ }^{1, b}$, Henry Hernández ${ }^{1,2, b}$, Carla Férnandez $1, a$
}

\begin{abstract}
RESUMEN
Entre 1995 a 2009 se registraron 206 hospitalizaciones en el Instituto Nacional de Salud del Niño debido a accidentes por mordedura canina. La mediana de edad de los pacientes fue cuatro años y para los días de hospitalización tres. El $61,7 \%$ fueron varones. Los accidentes ocurrieron en el domicilio de una tercera persona (39,3\%), en vía pública $(33,5 \%)$ y en el domicilio del agredido (27,2\%). El 66,5\% de los episodios fueron precedidos por un estímulo del niño y el $88,8 \%$ causado por animales reconocidos por los lesionados. El 34,0 \% reconoció la raza del animal $(52,9 \%$ animales cruzados). El 79,1 \% fueron lesiones múltiples y la región anatómica más afectada fueron la cabeza y cuello $(79,1 \%)$. Se registraron complicaciones en un $20,4 \%$ y secuelas estéticas en el $94,7 \%$. Las mordeduras caninas afectan la salud física de los niños.
\end{abstract}

Palabras clave: Accidentes; Perros; Niño; Vacunas antirrábicas (fuente: DeCS BIREME).

\section{DOG BITE ACCIDENTS IN A CHILDREN HOSPITAL AT LIMA, PERU. RETROSPECTIVE STUDY FROM 1995 - 2009}

\begin{abstract}
From 1995 to 2009, 206 cases of dog bites were recorded among hospitalized patients at the Children National Health of, Lima-Peru. The median of age was 4 years, and for hospitalization time was 3 days. Most patients were males $(61.7 \%)$. The bites occurred in the house of a third person (39.3\%), on the street (33.5\%), or in the victim's house (27.2 \%). A $66.5 \%$ of the bites were provoked and $88.8 \%$ were from animals known to the victim. The dog breed was recognized in $34 \%$ (52.9\% were mongrel dogs). Multiple lesions were identified in $79.1 \%$, and the most frequently bitten areas were head and neck (79.1\%). Complications were reported in $20.4 \%$ and aesthetic sequelae in $94.7 \%$.
\end{abstract}

Key words: Accidents; Dogs; Child; Rabies vaccines (source: MesH NLM).

\section{INTRODUCCIÓN}

Los accidentes por mordedura canina en personas representan un importante problema de salud pública, tanto por la lesión producida, como por el impacto emocional en las víctimas, las secuelas estéticas, la transmisión de enfermedades, las complicaciones secundarias (infecciones bacterianas) y el costo que incurre para la comunidad y el estado ${ }^{(1,2)}$. En el caso de los niños, ellos se exponen a estos accidentes debido a que comparten espacios comunes con estos animales ${ }^{(3)}$ y porque a su edad, no interpretan eficientemente las señales previas de amenaza, como gruñidos o ladridos que pueden conducir a una agresión ${ }^{(4)}$.
El comportamiento de los perros es el resultado de la combinación de aspectos como su genética, trato, entrenamiento y socialización, aunque existen algunas razas potencialmente más peligrosas. Así, en Estados Unidos, los perros de raza rottweiler y pitbull son causantes de alrededor del $60 \%$ de las fatalidades debido a accidentes por mordedura ${ }^{(5)}$. La implementación de una legislación para prohibir la crianza de perros de razas peligrosas en muchos países no se ha investigado con profundidad, a excepción de un estudio realizado en el Reino Unido en 1990 que describió que tales políticas no tuvieron el impacto esperado ${ }^{(6)}$.

\footnotetext{
Grupo de Salud Pública Veterinaria, Facultad de Veterinaria y Zootecnia, Universidad Peruana Cayetano Heredia. Lima, Perú.

Oficina de Epidemiologia, Instituto Nacional de Salud del Niño. Lima, Perú.

Medico Veterinario Zootecnista; ${ }^{\text {b }}$ Médico Veterinario.

* Este estudio fue presentado como tesis para optar el titulo profesional de Médico Veterinario Zootecnista en la Facultad de Veterinaria y Zootecnia de la Universidad Peruana Cayetano Heredia en 2010.
} 
Aunque es imposible prevenir todas las mordeduras de perros, estas pueden reducirse en número ${ }^{(7)}$. Además, que una evaluación rápida y un tratamiento oportuno pueden prevenir las complicaciones de la herida ${ }^{(8)} \mathrm{y}$ minimizar el estrés postraumático que suele sobrevenir después del evento ${ }^{(9)}$.

En el Perú, la información acerca de accidentes por mordedura en niños no se encuentra sistematizada. Por ello, el objetivo del estudio fue determinar las características clínico-epidemiológicas de los casos hospitalizados de accidentes por mordedura en un hospital de niños en el periodo de 1995 - 2009, en la ciudad de Lima, Perú.

\section{EL ESTUDIO}

Estudio observacional, descriptivo retrospectivo que se realizó en el Instituto Nacional de Salud del Niño (INSN), ubicado en el distrito de Breña, Lima - Perú, entre los meses agosto de 2009 y marzo de 2010. El cual se basó en la recolección de información de accidentes por mordedura canina en el periodo 1995 - 2009.

La búsqueda de información incluyó la revisión de la base de datos virtual de la Oficina de Epidemiología del INSN, los registros de historias clínicas (epicrisis e historias completas) y las placas radiográficas de los pacientes hospitalizados durante el periodo de estudio. La información relacionada con el evento de la mordedura se registra como parte del protocolo de atención al paciente (registro de atención por mordedura canina - reporte de casos).

Para la recolección de información se elaboró una ficha de datos. Dicho documento incluía la siguiente

Tabla 1. Distribución de los casos de mordedura canina según edad y circunstancia del accidente en relación al lugar del evento, Instituto Nacional de Salud del Niño (1995 - 2009).

\begin{tabular}{|c|c|c|c|c|}
\hline & \multicolumn{3}{|c|}{ Lugar del accidente } & \multirow{2}{*}{ TOTAL } \\
\hline & $\begin{array}{c}\text { Vía } \\
\text { pública }\end{array}$ & $\begin{array}{l}\text { Domicilio } \\
\text { de tercero }\end{array}$ & $\begin{array}{c}\text { Domicilio } \\
\text { del agredido }\end{array}$ & \\
\hline & N (\%) & N (\%) & $\mathrm{N}(\%)$ & N ( \%) \\
\hline \multicolumn{5}{|l|}{ Edad } \\
\hline 0 a 4 años & $31(15,0)$ & $49(23,8)$ & $37(18,0)$ & $117(56,8)$ \\
\hline 5 a 9 años & $24(11,7)$ & $25(12,1)$ & $15(7,3)$ & $64(31,1)$ \\
\hline 10 a 14 años & $14(6,8)$ & $7(3,4)$ & $4(1,9)$ & $25(12,1)$ \\
\hline \multicolumn{5}{|c|}{ Circunstancia del accidente } \\
\hline Sorpresivo & $48(23,3)$ & $14(6,8)$ & $7(3,4)$ & $69(33,5)$ \\
\hline Provocado & $21(10,2)$ & $67(32,5)$ & $49(23,8)$ & $137(66,5)$ \\
\hline TOTAL & $69(33,5)$ & $81(39,3)$ & $56(27,2)$ & $206(100)$ \\
\hline
\end{tabular}

información: datos generales del paciente agredido (sexo, grupo de edad, procedencia, tiempo de estancia hospitalaria), variables del entorno (lugar y circunstancia del accidente), del animal agresor (raza, condición de tenencia y propiedad) y las relacionadas a la lesión (número, tipo de exposición, profundidad, ubicación anatómica, tratamientos, secuelas y complicaciones).

La información obtenida fue transferida a una base de datos en el programa Microsoft Excel y se utilizó el programa SPSS 15.0 para su análisis utilizando estadística descriptiva. Las variables cuantitativas, edad del paciente y tiempo de hospitalización, se resumieron utilizando la mediana como medidas de tendencia central. El resto de resultados se muestran en tablas de frecuencias.

La Oficina Ejecutiva de Apoyo a la Investigación y Docencia Especializada del INSN autorizó la presente investigación (código E-12/09).

\section{HALLAZGOS}

Para el presente estudio, se incluyó al total de niños (206) hospitalizados por mordedura canina durante el periodo 1995 - 2009. La distribución de los accidentados según edad fue: 56,8 \% (117) menores de cuatro años, 31,1\% (64) de cinco a nueve años y 12,1 \% (25) mayores de diez años, siendo la mediana de edad de cuatro años. El 61,7\% (127) fueron varones y 38,3 \% (79) mujeres. El $83 \%$ (171) procedieron de zona urbana, 9,7\% (20) de zona semiurbana y $7,3 \%$ (15) de zona rural. El tiempo de hospitalización fue de un día para el 30,6 \% (63) de los accidentados, 43,2 \% (89) entre dos y siete días, $23,8 \%$ (49) entre 8 a 30 días y 2,4 \% (5) fueron hospitalizados más de un mes, con una mediana de tres días. El 39,3 \% (81) de accidentes se presentó en el domicilio de una tercera persona; 33,5 \% (69) en la vía pública y $27,2 \%$ (56) en el domicilio del agredido. En el grupo de niños menores de cuatro años predominaron los accidentes en casa de un tercero, mientras que en el grupo de diez a catorce años la mayoría de casos se produjeron en la vía pública (Tabla 1).

El 66,5\% (137) de los accidentes fueron precedidos por un estímulo del niño (evento provocado) y 33,5\% (69) fueron ataques intempestivos del animal (evento sorpresivo). Los accidentes sorpresivos fueron más frecuentes en la vía pública, mientras que los provocados ocurrieron en la casa de un tercero (Tabla 1). El 88,8 \% (183) fueron causados por animales reconocidos por los lesionados. Se registró la raza del animal en el $34 \%$ (70) de los accidentes. De ellos, el 52,9 \% (37) correspondió a razas cruzadas y la diferencia a razas definidas (pitbull, rottweiler y bóxer, entre otras). 
El 20,9\% (43) de lesiones fueron únicas y 79,1\% (163) fueron múltiples. Se registró en total 327 lesiones, siendo la región de cabeza y cuello la más afectada (Tabla 2). El 92,7\% (191) de los accidentados sufrieron lesiones profundas y la exposición fue considerada grave en el $97,6 \%$ (201) de los casos.

Al 78,6 \% (162) se le administró el esquema preventivo de vacunación antirrábica (vacunar hasta el quinto día posmordedura), al 8,7 \% (18) el esquema reducido y $12,6 \%$ (26) no requirieron vacunación. El 96,1 \% (198) de los casos recibieron tratamiento antibiótico siendo la penicilina (oxacilina) más ácido clavulánico los usados con mayor frecuencia.

El 20,4 \% (42) de pacientes presentó complicaciones como infecciones bacterianas, necrosis, problemas genitourinarios (amputación traumática del miembro debido a la mordedura, disuria, postitis, retención urinaria, edema de la zona afectada, necrosis posterior

Tabla 2. Ubicaciones anatómicas de las mordeduras caninas en pacientes hospitalizados en el "Instituto Nacional de Salud del Niño". Período 1995 - 2009, Lima, Perú.

\begin{tabular}{|c|c|c|}
\hline Ubicación & $\mathbf{N}$ & $(\%)$ \\
\hline \multicolumn{3}{|l|}{ Cabeza y cuello } \\
\hline Cara (frente y mejillas) & 126 & $(38,5)$ \\
\hline Labio & 40 & $(12,2)$ \\
\hline Nariz & 18 & $(5,6)$ \\
\hline Cráneo & 17 & $(5,2)$ \\
\hline Globo ocular & 12 & $(3,7)$ \\
\hline Oreja & 12 & $(3,7)$ \\
\hline Lagrimal & 9 & $(2,8)$ \\
\hline Cuello & 5 & $(1,5)$ \\
\hline Lengua (y paladar blando) & 2 & $(0,6)$ \\
\hline \multicolumn{3}{|l|}{ Miembro superior } \\
\hline Brazo & 11 & $(3,4)$ \\
\hline Antebrazo & 11 & $(3,4)$ \\
\hline Hombro & 5 & $(1,5)$ \\
\hline Dedos de la mano & 5 & $(1,5)$ \\
\hline Mano & 5 & $(1,5)$ \\
\hline Codo & 1 & $(0,3)$ \\
\hline \multicolumn{3}{|l|}{ Miembro inferior } \\
\hline Pierna & 17 & $(5,2)$ \\
\hline Muslo & 5 & $(1,5)$ \\
\hline Pie & 3 & $(0,9)$ \\
\hline Rodilla & 1 & $(0,3)$ \\
\hline \multicolumn{3}{|l|}{ Genitales/Región anal } \\
\hline Genitales & 10 & $(3,1)$ \\
\hline Glúteo & 4 & $(1,2)$ \\
\hline Región anal & 1 & $(0,3)$ \\
\hline Tórax & 5 & $(1,5)$ \\
\hline Abdomen & 2 & $(0,6)$ \\
\hline TOTAL & 327 & $(100,0)$ \\
\hline
\end{tabular}

a la cirugía) como consecuencia de la mordedura. El $94,7 \%$ (195) tuvieron secuelas del tipo físico. De ellos, las cicatrices en la zona de lesión se presentó en el $96,4 \%$ (188) de los casos, amputaciones en el 2,6\% (5) y en el $1 \%$ (2) de los pacientes presentaron ambas complicaciones.

\section{DISCUSIÓN}

La casuística mostrada representaría solo una parte de estos accidentes que se habrían producido en niños durante el periodo de estudio. Se estima que solo el $25 \%$ de agresiones se registran, ello se debe a la gravedad de la lesión producida ${ }^{(10)}$. El que los varones sean los más afectados se debería a que ellos suelen compartir espacios en común y realizan actividades más bruscas o temerarias con los animales, lo que podría ser el desencadenante de una agresión canina $(4,11,12)$. La edad más afectada fue hasta los cuatro años, lo que estaría asociado al desconocimiento de las conductas que pueden provocar al animal, la incapacidad de advertir a tiempo las actitudes amenazantes, el poseer menos coordinación y fuerza muscular y porque no son capaces de defenderse con sus extremidades durante un ataque.

Las agresiones causadas por un animal conocido se deberían a que los niños no consideran a estos como peligrosos y tratan de jugar con ellos, provocando la agresión. Generalmente ocurren en domicilios (hogar propio o el de un familiar o amigo) y en menores bajo custodia de los padres, quienes no advierten la presencia de estas mascotas como un peligro. En contraste, en la vía pública, las personas tienden a evitar el contacto con animales desconocidos o sin dueño, por lo que los accidentes suelen ser sorpresivos. Estos son más frecuentes en niños mayores a diez años, quienes por su edad tienen más probabilidad de encontrarse fuera del hogar (vía pública) especialmente en horas de juego.

Aunque existe un subregistro acerca de la raza del animal involucrado en accidentes por mordedura, ello no resultaría relevante si se considera que la agresividad mostrada por los animales surge principalmente por la tenencia irresponsable de sus dueños ${ }^{(13)}$.

La ubicación anatómica afectada mayormente fue la región de la cabeza y cuello. La importancia de ellas radica en el riesgo de presentarse fracturas ocultas de huesos craneales y por que las lesiones localizadas en el rostro son desfigurantes y psicológicamente peor aceptadas ${ }^{(14)}$. La mayor parte de mordeduras fueron de tipo profundo y grave, requiriendo tratamiento o alguna intervención quirúrgica. El predominio de lesiones 
múltiple estaría relacionado con la limitada capacidad de defensa por parte de la víctima durante la agresión, resultando como consecuencia una alta frecuencia de pacientes con cicatrices como secuela física.

La administración de antibiótico como tratamiento se ha justificado por considerar que toda herida causada por mordedura estaría contaminada por la flora polimicrobiana que se encuentra en la cavidad oral del can. En atención a la Norma técnica de salud para la prevención y control de rabia humana en el Perú (2007) y debido a que la mayoría de agresiones fueron producidas por animales conocidos sin signos de rabia, la aplicación de la vacunación antirrábica preventiva (hasta el día cinco posmordedura) fue el esquema más utilizado ${ }^{(15)}$.

En conclusión, las características epidemiológicas y clínicas de los accidentes por mordeduras caninas las convierten en un problema de salud pública que afectan la salud física y psicológica de los niños, por lo que se requiere la atención, especialmente preventiva, de las autoridades de salud.

\section{Contribuciones de autoría}

$\mathrm{HH}, \mathrm{CM}$ y NF participaron en la concepción y diseño del trabajo. $\mathrm{HH}$ y $\mathrm{CM}$ participaron en la recolección/ obtención de resultados, NF y CF participaron en el análisis e interpretación de datos, CM, NF y CF participaron en la redacción del manuscrito. NF participó en la asesoría estadística y análisis de datos. CM y CF participaron en la obtención del financiamiento. Todos los autores revisaron en forma crítica versiones preliminares del manuscrito y aprobaron la versión final del trabajo.

\section{Fuentes de financiamiento}

Beca Estimulo "Bárbara Ann Kotowski De Tejada". Código SIDISI N. ${ }^{\circ}$ 55930. Dirección Universitaria de Investigación, Ciencia y Tecnología - Universidad Peruana Cayetano Heredia.

\section{Conflictos de interés}

Los autores declaran no tener conflictos de interés en la publicación de este artículo.

\section{REFERENCIAS BIBLIOGRÁFICAS}

1. Glausiuss G, Ascione I, Sehabiague G. Mordeduras por animales en la edad pediátrica. Arch Pediatr Urug. 2000;71:24-30.

2. AVMA American Veterinary Medical Association. A community approach to dog bite prevention. JAVMA. 2001;218(11):1732-49.

3. Navia M. Mordedura de perro en cara. Arch Argent Pediatr. 2005;103(5):396-400.

4. Lema F. Mordedura de perro, comportamiento y agresión. Arch. Argent. Pediatr. 2005;103(5):387-8.

5. Sacks J, Sinclair L, Glichrist J, Colab G, Lockwood R. Breeds of dogs involved in fatal human attacks in the United States between 1979 and 1998. JAVMA. 2000:217(6):836-40.

6. Daniels D, Rovane B, Ritzi B, O’Neil J. Analysis of nonfatal dog bites in children. J Trauma. 2009;66(3 Suppl):S17-22.

7. Feldman K, Trent R, Jay M. Epidemiology of hospitalizations resulting from dog bites in California, 1991-1998. Am J Public Health. 2004;94(11):1940-1.

8. Abuabara A. A review of facial injuries due to dog bites. Med Oral Patol Oral Cir Bucal. 2006;11:348-50.

9. Gomes F, Malago W, Cardeal1 D, Bor-Seng-Shu E, Ferreira de Andrade A, Jacobsen M. Craniocerebral injuries from dog bites. Arq Neuropsiquiatr 2008;66(2B):397-9.

10. Méndez R, Gómez M, Somoza I, Liras J, Pais E, Vela D. Mordeduras de perro. Análisis de 654 casos en 10 años. An Esp Pediatr. 2002;56:425-9.

11. Zanini F, Padinger P, Elissondo M, Perez H. Epidemiología de las lesiones por mordedura de perro en Tierra del Fuego, Argentina. Medicina (B. Aires). 2008;68:1-5

12. Hernández D. Mordedura de perro: enfoque epidemiológico de las lesiones causadas por mordedura de perro. Revista de Enfermedades Infecciosas de Pediatría. 2009;23(89):13-20.

13. Schvartzman S, Pacín M. Lesiones por mordedura de perros en niños. Arch Argent Pediatr. 2005;103(5):389-95.

14. Álvez F. Infecciones por mordeduras y heridas punzantes. En: Asociación Española de Pediatría. Protocolos Diagnóstico Terapéuticos de la AEP: Infectología Pediátrica. Madrid: AEP; 2008. p. 161-71.

15. López R. Reemergencia de la rabia en el Perú. Rev Peru Med Exp Salud Publica. 2007;24(1):3-4.

Correspondencia: Néstor Falcón Pérez

Dirección: Av. Honorio Delgado 430, Lima 31, Perú.

Teléfono: (511) 3190000 anexo 3001 / 996533579

Correo electrónico: nestor.falcon@upch.pe 